\title{
Brain Stem Infarction Due to Basilar Artery Dissection in a Patient with a Parotitis and Hyperhomocysteinemia
}

\author{
Byoung June Ahn \\ Department of Neurology, Soonchunhyang University Gumi Hospital, Gumi, Korea
}

\begin{abstract}
A 29-year-old man developed sudden dysarthria and right-sided weakness 3 days before admission to hospital. He was diagnosed with parotitis in the emergency room of Soonchunhyang University Gumi Hospital. The plasma levels of homocysteine were elevated $(30.48 \mathrm{mg} / \mathrm{dL})$. A brain magnetic resonance imaging scan revealed a high signal intensity lesion in the left paramedian pons and computed tomography angiography showed a marked narrowing of the mid-basilar artery. We report a rare case of spontaneous basilar artery dissection caused by a recent infection and hyperhomocysteinemia.
\end{abstract}

Keywords: Basilar artery dissection; Parotitis; Hyperhomocysteinemi

\section{INTRODUCTION}

Isolated dissection of the basilar artery is rare. The pathogenesis of arterial dissection is unknown. Trauma and primary disease of the arterial wall are well known predisposing factors. Recent infection and mild Hyperhomocysteinemia can be another independent risk factor.

\section{CASE REPORT}

A 29-year-old man was admitted to Soonchunhyang University Gumi Hospital with the presentation of weakness on the right side and dysarthria, with duration of 3 days preceding the day of admission. He was diagnosed with parotitis in our emergency room. The common cardiovascular risk factors such as hypertension, diabetes mellitus, and hyperlipidemia were absent. On initial examination, the patient was alert and oriented. He was afebrile, with a blood pressure of 130/80 $\mathrm{mm} \mathrm{Hg}$, and a pulse rate of 80 beats per minute. The neurological examination revealed hemiparesis on the right side (Medical Research Council scale grade 4/5). The plantar response was normal on the left side, but was up-going on the right side. The sensory and cerebellar functions were normal. The pupillary light reflexes were normal bilaterally. The results of the following tests were unremarkable: electrocardiogram, complete blood cell count, erythrocyte sedimentation rate, blood sugar, lipid profile, fibrinogen degradation products, prothrombin time, lupus anticoagulant, antithrombin III, antiphospholipid antibody, anticardiolipin antibody, antinuclear antibody, protein C, protein S, C3, and C4. However, the plasma levels of homocysteine were elevated (30.48 mg/dL). A serum DNA analysis of methylenetetrahydrofolate reductase (MTHFR) was also done. The result revealed homozygosity for MTHFR deficiency. A brain magnetic resonance imaging scan revealed a high signal intensity lesion in the left paramedian pons (Fig. 1). We also performed computed tomography angiography (CTA) that showed a marked narrowing of the mid-basilar artery; the axial image showed an intramural hematoma adjacent to the narrowed basilar artery (Fig. 2). The patient was treated with anticoagulation therapy. He was discharged with only mild right-leg weakness. A follow-up brain CTA was performed after five months that showed no abnormal lesions (Fig. 3). We report a rare case of spontaneous basilar artery dissection associated with a recent infection and hyperhomocysteinemia. 


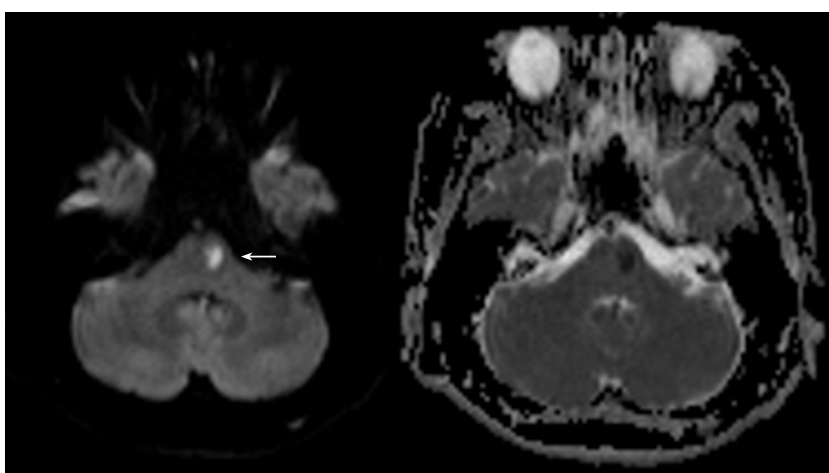

Fig. 1. Diffusion-weighted magnetic resonance imaging showing high signal intensity lesions (arrow) within left paramedian pons.
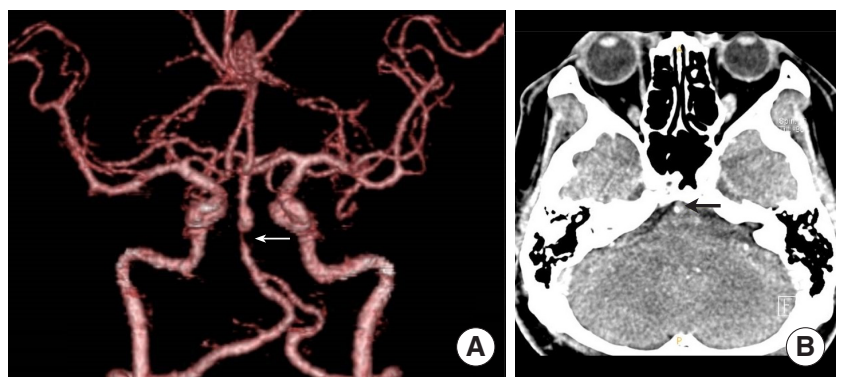

Fig. 2. (A) Computed tomography angiogram showing marked narrowing (white arrow) of the basilar artery and (B) axial image showing intramural hematoma (black arrow) adjacent to a narrowed basilar artery.

\section{DISCUSSION}

Isolated basilar artery dissection is a rare disease. The basilar artery is usually involved by extension of distal vertebral artery dissection [1]. Its various possible clinical manifestations range from brain stem infarction to subarachnoid hemorrhage. Rare cases of spontaneous basilar artery dissection in previously healthy people have been described. Most cases have occurred in older patients with basilar artery atherosclerosis. The predisposing factors for basilar artery dissection include trauma, atherosclerosis, hypertension, cystic medial degeneration, fibromuscular dysplasia, syphilis, contraceptive drugs, and migraine. Spontaneous arterial dissection is a major cause of strokes in young adults. Constitutional and environmental factors may also be involved. Among the environmental factors, a recent infection has been suggested as a potential triggering factor by Grau et al. [2] and Somer and Finegold [3]. The inflammation during the infections and the microbial agents themselves can cause substantial damage to the vascular walls. During infection proinflammatory cytokines, free radicals, and proteases can damage the vessel wall $[4,5]$.
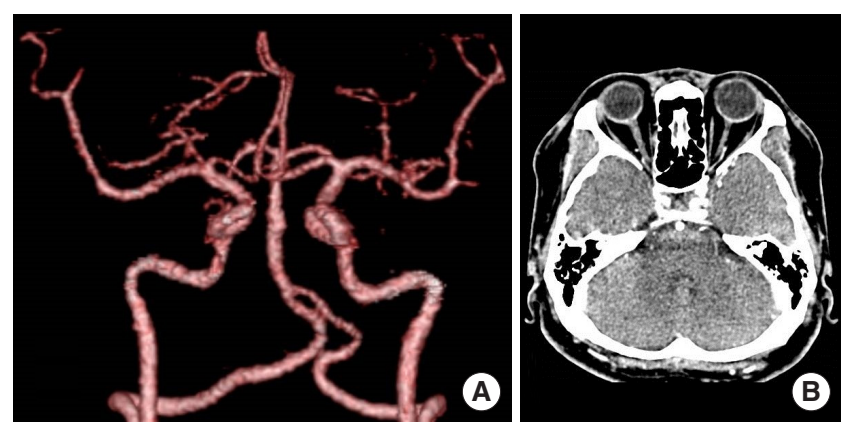

Fig. 3. (A) Follow-up computed tomography angiogram showing improved vessel wall of the basilar artery and $(B)$ axial image showing intramural hematoma resolved.

Hyperhomocysteinemia may be another risk factor. Homocysteine is a multipotent molecule that can injure the endothelium through various mechanisms. It facilitates oxidative arterial injury, damages the vascular matrix, and induces vascular smooth muscle proliferation [6,7]. The importance of hyperhomocysteinemia in inducing endothelial damage is well documented by different in vitro and in vivo studies. Woo et al. [8] have demonstrated that in patients with hyperhomocysteinemia there is an impaired endothelium-dependent flow-mediated vasodilation. Our patient demonstrated homozygosity for MTHFR deficiency that leads to a less severe form of hyperhomocysteinemia. Arauz et al. [9] found that mild hyperhomocysteinemia and low folate levels were highly associated with an increased risk of cervical arterial dissection. Already existed cerebral artery dissection could be suggested. But given his age and history, that possibility is less likely. We suppose that hyperhomocysteinemia and recent infection could interact in the cascade of pathogenetic events leading to arterial wall damage with additive effect.

\section{REFERENCES}

1. Mokri B, Houser OW, Sandok BA, Piepgras DG. Spontaneous dissections of the vertebral arteries. Neurology 1988;38:880-5.

2. Grau AJ, Brandt T, Buggle F, Orberk E, Mytilineos J, Werle E, et al. Association of cervical artery dissection with recent infection. Arch Neurol 1999;56:851-6.

3. Somer T, Finegold SM. Vasculitides associated with infections, immunization, and antimicrobial drugs. Clin Infect Dis 1995;20:1010-36.

4. Smedly LA, Tonnesen MG, Sandhaus RA, Haslett C, Guthrie LA, Johnston RB Jr, et al. Neutrophil-mediated injury to endothelial cells: enhancement by endotoxin and essential role of neutrophil elastase. J Clin Invest 1986;77:1233-43.

5. Pober JS. Warner-Lambert/Parke-Davis award lecture: cytokine-mediated activation of vascular endothelium: physiology and pathology. Am J Pathol 1988;133:426-33. 
6. Tsai JC, Perrella MA, Yoshizumi M, Hsieh CM, Haber E, Schlegel R, et al. Promotion of vascular smooth muscle cell growth by homocysteine: a link to atherosclerosis. Proc Natl Acad Sci U S A 1994;91:6369-73.

7. Lang D, Kredan MB, Moat SJ, Hussain SA, Powell CA, Bellamy MF, et al. Homocysteine-induced inhibition of endothelium-dependent relaxation in rabbit aorta: role for superoxide anions. Arterioscler Thromb Vasc Biol 2000;20:422-7.
8. Woo KS, Chook P, Lolin YI, Cheung AS, Chan LT, Sun YY, et al. Hyperhomocyst(e)inemia is a risk factor for arterial endothelial dysfunction in humans. Circulation 1997;96:2542-4.

9. Arauz A, Hoyos L, Cantu C, Jara A, Martinez L, Garcia I, et al. Mild hyperhomocysteinemia and low folate concentrations as risk factors for cervical arterial dissection. Cerebrovasc Dis 2007;24:210-4. 